\title{
Scattering by a toroidal coil
}

\author{
Ph. Roux ${ }^{\dagger}$ \\ Department of Mathematics, University of Rennes, \\ Campus Beaulieu, 35042, Rennes, France, \\ Philippe.Roux@univ-rennes1.fr
}

\begin{abstract}
In this paper we consider the Schrödinger operator in $\mathbb{R}^{3}$ with a long-range magnetic potential associated to a magnetic field supported inside a torus $\mathbb{T}$. Using the scheme of smooth perturbations we construct stationary modified wave operators and the corresponding scattering matrix $S(\lambda)$. We prove that the essential spectrum of $S(\lambda)$ is an interval of the unit circle depending only on the magnetic flux $\phi$ across the section of $\mathbb{T}$. Additionally we show that, in contrast to the Aharonov-Bohm potential in $\mathbb{R}^{2}$, the total scattering cross-section is always finite. We also conjecture that the case treated here is a typical example in dimension 3.
\end{abstract}

PACS numbers : 03.65.N, 11.55, 72.15.N, MSC : 35P25, 81U05, $81 \mathrm{U} 20$

\section{Introduction}

Let $A(x)$ be a magnetic potential

$$
A(x)=\frac{a\left(\varphi_{x}\right)}{|x|} \mathbf{e}_{\varphi_{x}}, \quad|x| \geq R>0
$$

\footnotetext{
${ }^{\dagger}$ permanent address : IUT Lannion, Département Informatique, rue Branly BP150, 22302 Lannion CEDEX
} 
where $a \in C_{0}^{\infty}(0, \pi)$ is a positive function of the colatitude of $x=\left(x_{1}, x_{2}, x_{3}\right)$

$$
\varphi_{x}=\arccos \left(\frac{x_{3}}{|x|}\right) \in[0, \pi],
$$

and $\mathbf{e}_{\varphi_{x}}$ denotes the unitary vector of spherical coordinates $\left(\mathbf{e}_{r_{x}}, \mathbf{e}_{\varphi_{x}}, \mathbf{e}_{\theta_{x}}\right)$ associated to the point $x$

$$
\mathbf{e}_{\varphi_{x}}=\frac{1}{|x|}\left(\frac{x_{1} x_{3}}{\sqrt{x_{1}^{2}+x_{2}^{2}}}, \frac{x_{2} x_{3}}{\sqrt{x_{1}^{2}+x_{2}^{2}}},-\sqrt{x_{1}^{2}+x_{2}^{2}}\right) .
$$

Physically the potential (1.1) corresponds to a magnetic field $B=\operatorname{curl} A$ supported inside a torus $\mathbb{T}$ obtained by revolution around the $x_{3}$ axis (see Fig. 11). The function $a$ in (1.1) depends only on the section of $\mathbb{T}$ and the flux of $B$ across this section is

$$
\phi=\int_{0}^{\pi} a(\varphi) d \varphi>0 .
$$

This situation is kwown, from the work of Aharonov and Bohm [AB59, to show a purely quantum phenomena : a compactly supported magnetic field can act on particles which never cross its support. From the mathematical point of view, despite $B$ as a finite support, the potential $A$ decays as $|x|^{-1}$ at infinity and is of long-range nature. Thus one expects that the properties of the scattering process associated to the potential (1.1) be different from the case of short-range potentials.

Here we consider the Schrödinger operator

$$
H=(D-A(x))^{2}, \quad D=-i \nabla_{x},
$$

in $\mathbb{L}^{2}\left(\mathbb{R}^{3}\right)$, and develop the scheme of smooth perturbations for the pair $H, H_{0}=-\Delta$. Although usual wave operators exists (due to the transversal gauge condition $\langle A(x), x\rangle=0$, for all $x \in \mathbb{R}^{3}$, see [LT87]) we prefer to work with modified wave operators of the Isozaki-Kitada type :

$$
W_{ \pm}\left(H, H_{0}, J\right)=s-\lim _{t \rightarrow \pm \infty} e^{i t H} J e^{-i t H_{0}}
$$

with stationary identifications $J=J_{ \pm}$depending on the sign of $t$, as in Nic94, RY02b. We choose the operators $J_{ \pm}$as pseudo-differential operators (PDO) with symbols $\exp \left(i \Phi_{ \pm}(x, \xi)\right)$ such that the effective perturbation 
$T_{ \pm}=H J_{ \pm}-J_{ \pm} H_{0}$ be short-range, that is the phase-function $\Phi_{ \pm}$satisfies $\nabla_{x} \Phi_{ \pm}(x, \xi)=A(x)$. Thus the existence of wave operators (1.6) relies only on the limiting absorption principle in contrast to [RY02b where the radiation estimate was needed. Since the identifications $J_{ \pm}$are "close" to unitary operators, the wave operators $W_{ \pm}\left(H, H_{0}, J_{ \pm}\right)$are automatically isometric and complete, indeed, they coincide with the usual wave operators $W_{ \pm}\left(H, H_{0}\right)=W_{ \pm}\left(H, H_{0}, I d\right)$.

The scattering operator, defined by $S=W_{+}^{*}\left(H, H_{0}\right) W_{-}\left(H, H_{0}\right)$, commutes with $H_{0}$, so, in the spectral representation of $H_{0}$, it reduces to the multiplication by the operator valued function $S(\lambda)$, named the scattering matrix (SM) which acts as an integral operator on the unit sphere $\mathbb{S}^{2}$ of $\mathbb{R}^{3}$. Our study of the SM relies on its stationary representation

$$
S(\lambda)=\mathcal{W}(\lambda)-2 i \pi \Gamma_{0}(\lambda)\left(J_{+}^{*} T_{-}-T_{+}^{*} R(\lambda+i 0) T_{-}\right) \Gamma_{0}^{*}(\lambda)
$$

where $R(z)=(H-z)^{-1}$, and

$$
\mathcal{W}(\lambda)=\Gamma_{0}(\lambda) W_{+}\left(H_{0}, H_{0}, J_{+}^{*} J_{-}\right) \Gamma_{0}^{*}(\lambda)
$$

with $\Gamma_{0}(\lambda): \mathbb{L}^{2}\left(\mathbb{R}^{3}\right) \longrightarrow \mathbb{L}^{2}\left(\mathbb{S}^{2}\right)$ defined for $u$ in the Schwarz class by

$$
\left(\Gamma_{0}(\lambda) u\right)(\omega)=\frac{\sqrt{\lambda}}{2(2 \pi)^{3 / 2}} \int_{\mathbb{R}^{3}} e^{i \sqrt{\lambda}<\omega, x>} u(x) d x, \quad \omega \in \mathbb{S}^{2},
$$

and $\Gamma_{0}^{*}(\lambda)$ is the formally adjoint to $\Gamma_{0}(\lambda)$. To justify the formula (1.7) we decompose it as a sum of bounded operators. First we calculate the term $\mathcal{W}(\lambda)$ and prove that it reduces to the operator of multiplication by the function w defined on $\mathbb{S}^{2}$ by

$$
\mathrm{w}(\omega)=\exp \left(i \int_{\pi-\varphi_{\omega}}^{\varphi_{\omega}} a(\varphi) d \varphi\right), \omega \in \mathbb{S}^{2} .
$$

Then we show that the remaining term, $S(\lambda)-\mathcal{W}(\lambda)$, is an integral operator on $\mathbb{S}^{2}$ with a $C^{\infty}$ kernel. Thus, we can make a spectral analysis of the SM. Since $S(\lambda)$ is a compact perturbation of $\mathcal{W}(\lambda)$, we calculate its essential spectrum, that is

$$
\sigma_{\text {ess }}(S(\lambda))=\{\mu=\exp (i \nu) \in \mathbb{C} \mid \nu \in[-\phi, \phi]\}
$$


In particular $\sigma_{\text {ess }}(\lambda)$ depends only on the magnetic flux $\phi$ (1.4) of $B$ across the section of $\mathbb{T}$. Now if we take as a definition of the differential scattering cross-section

$$
\Sigma_{d i f f}\left(\omega, \omega_{0} ; \lambda\right)=\frac{\lambda^{(-d-1) / 2}}{(2 \pi)^{d-1}}\left|\mathrm{~s}\left(\omega, \omega_{0} ; \lambda\right)\right|^{2}, \quad \omega \neq \omega_{0},
$$

with $d=3$ and where $\omega_{0}$ (resp. $\omega$ ) is the in-coming (out-going) direction, then the function $\Sigma_{\text {diff }}\left(\omega, \omega_{0} ; \lambda\right)$ belongs to $C^{\infty}\left(\mathbb{S}^{2} \times \mathbb{S}^{2} \times \mathbb{R}^{+}\right)$. In particular the total scattering cross-section

$$
\Sigma_{t o t}\left(\omega_{0} ; \lambda\right)=\int_{\mathbb{S}^{2}} \Sigma_{d i f f}\left(\omega, \omega_{0} ; \lambda\right) d \omega
$$

is finite for all incident directions $\omega_{0} \in \mathbb{S}^{2}$.

The paper is organized as follow : in section 2 we construct stationary wave operators and recover the basic results of scattering theory for potential (1.1); in section 3 we analyze the structure of the SM and its spectral properties; finally, in section 4 we make some remarks about this example

and the 2-dimensional Aharonov-Bohm effect, we also conjecture that the situation described here is very general in the 3-dimensional case.

\section{Wave operators}

In this section we construct time-independant modified wave operators, as in Nic94, RY02b, and recover basic results on long-range magnetic scattering in transversal gauge [LT87.

2.1. In the scheme of smooth perturbations the choice of identifications $J=J_{ \pm}$in (1.6) is determined by the condition that the effective perturbation $T_{ \pm}=H J_{ \pm}-J_{ \pm} H_{0}$ be "short-range". If, as in Yaf98, we search $J_{ \pm}$as a PDO with symbol $j_{ \pm}(x, \xi)$ then the function $\Psi_{ \pm}(x, \xi)=e^{i<x, \xi>} j_{ \pm}(x, \xi)$ should be an approximate (i.e. up to short-range terms) eigenfunction of $H$ assiociated to the eigenvalue $|\xi|^{2}$. Thus we set $j_{ \pm}(x, \xi)=\exp \left(i \Phi_{ \pm}(x, \xi)\right)$ and compute

$$
\begin{aligned}
\left(H-|\xi|^{2}\right) \Psi_{ \pm}(x, \xi)=( & 2<\xi, \nabla_{x} \Phi_{ \pm}(x, \xi)-A(x)>+\left|\nabla_{x} \Phi_{ \pm}(x, \xi)-A(x)\right|^{2} \\
& \left.-i \operatorname{div}_{x}\left(\nabla_{x} \Phi_{ \pm}(x, \xi)-A(x)\right)\right) \Psi_{ \pm}(x, \xi)
\end{aligned}
$$


Taking only the principal (i.e. the first) term of (2.11), we obtain the eikonal equation for $\Phi_{ \pm}$

$$
<\xi, \nabla_{x} \Phi_{ \pm}(x, \xi)-A(x)>=0 .
$$

As shown in [Yaf98, this equation admits solutions with decaying derivatives for large $|x|$

$\Phi_{ \pm}(x, \xi)=\mp \int_{0}^{\infty}<A(x \pm t \xi)-A( \pm t \xi), \xi>d t=\mp \int_{0}^{\infty}<A(x \pm t \xi), \xi>d t$.

Note that the second equality is a consequence of the transversal gauge condition $\langle A(y), y\rangle=0$, for all $y \in \mathbb{R}^{3}$. To simplify this expression we first make the change of variables $t \mapsto s$ defined by

$$
s=s_{0} \pm t|\xi|, \quad x=b+s_{0} \omega, \quad<b, \omega>=0, \quad \omega=\frac{\xi}{|\xi|},
$$

which leads to the equation

$$
\Phi_{ \pm}(x, \xi)=\int_{ \pm \infty}^{s_{0}}<A(b+s \omega), \omega>d s .
$$

Then, we rewrite this integral into spherical coordinates (see Fig. I). Let

$$
x(s)=b+s \omega, \quad u(s)=\sqrt{\left(b_{1}+s \omega_{1}\right)^{2}+\left(b_{2}+s \omega_{2}\right)^{2}},
$$

and $\varphi(s)$ be the colatitude of $x(s)$ (defined by (1.3)). Since

$$
\sin (\varphi(s))=u(s) /|x(s)|, \quad \cos (\varphi(s))=\left(b_{3}+s \omega_{3}\right) /|x(s)|,
$$

and taking into account that

$$
|x|^{2}=|b|^{2}+s^{2},|\omega|^{2}=1, \quad<x(s), \omega>=s, \frac{d}{d s} \cos (\varphi(s))=-\sin (\varphi(s)) \frac{d \varphi(s)}{d s},
$$

we get

$$
\frac{d \varphi(s)}{d s}=\frac{s b_{3}-\omega_{3}|b|^{2}}{|x(s)|^{2} u(s)}
$$

On the other hand

$$
\begin{aligned}
<\mathbf{e}_{\varphi(s)}, \omega> & =\frac{1}{|x(s)| u(s)}\left(x_{3}(s)<x(s), \omega>-<\left(0,0,|x(s)|^{2}\right), \omega>\right) \\
& =\frac{|x(s)|^{2} \omega_{3}-s x_{3}(s)}{|x(s)| u(s)}=\frac{s b_{3}-|b|^{2} \omega_{3}}{|x(s)| u(s)}
\end{aligned}
$$


which leads to

$$
\begin{aligned}
<A(x(s)), \omega> & =\frac{a(\varphi(s))}{|x(s)|}<\mathbf{e}_{\varphi(s)}, \omega> \\
& =\frac{a(\varphi(s))}{|x(s)|} \frac{s b_{3}-|b|^{2} \omega_{3}}{|x(s)| u(s)}=a(\varphi(s)) \frac{d \varphi(s)}{d s} .
\end{aligned}
$$

Thus, we can make the change of variables $s \mapsto \varphi(s)$ in (2.3) and, since $\varphi\left(s_{0}\right)=\varphi_{x}$ and $\varphi( \pm \infty)=\varphi_{ \pm \omega}=\varphi_{ \pm \xi}$, we get

$$
\Phi_{ \pm}(x, \xi)=\int_{\varphi_{ \pm \xi}}^{\varphi_{x}} a(\varphi) d \varphi, \quad|x| \geq R>0
$$

in particular, for $|x| \geq R>0$,

$$
\nabla_{x} \Phi_{ \pm}(x, \xi)=\frac{1}{|x|} \partial_{\varphi_{x}} \Phi_{ \pm}(x, \xi) \mathbf{e}_{\varphi_{x}}=A(x) .
$$

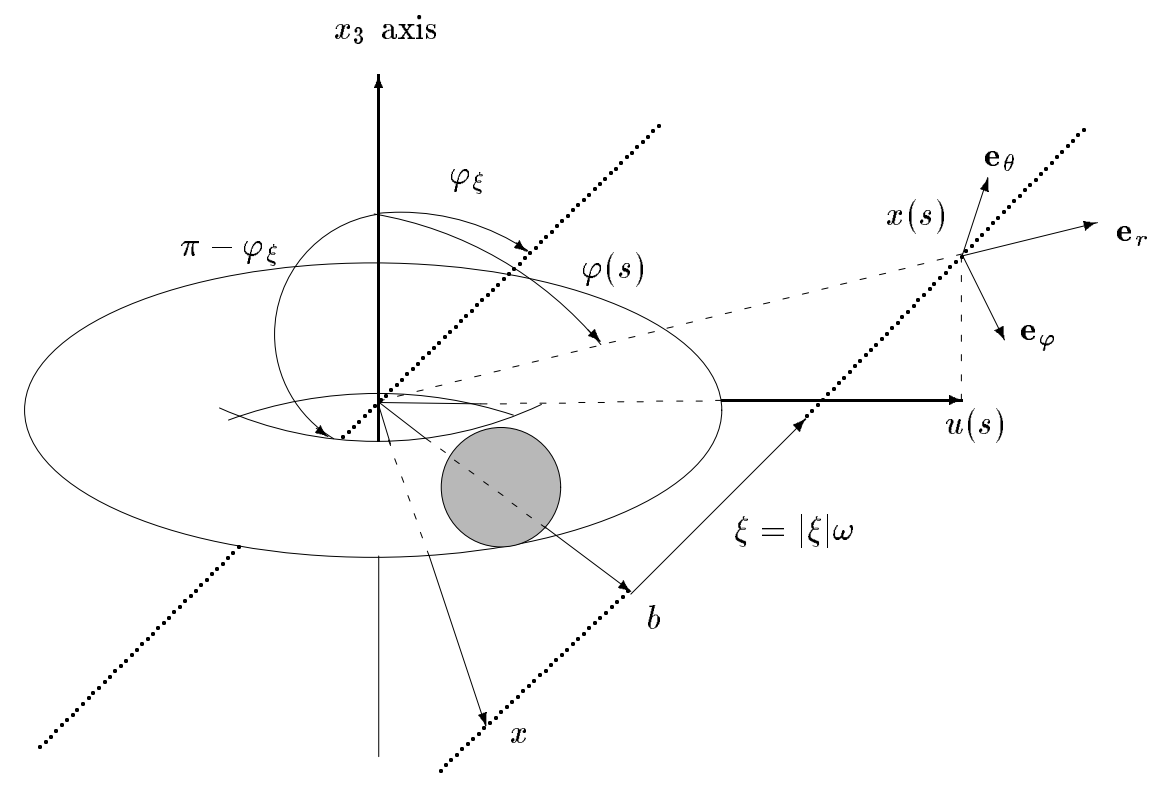

Figure 1: the toroidal coil $\mathbb{T}$

The stationary scheme developped below make an intensive use of symbolic calculus (see Tay81]), so we have to fix some notations on PDO. In the 
following we call $\mathcal{S}^{m}(\mu)$ the set of functions $p \in C^{\infty}\left(\mathbb{R}^{6}\right)$ satisfying, for all multi-indices $\alpha$ and $\beta$, the estimates

$$
\left|\partial_{x}^{\alpha} \partial_{\xi}^{\beta} p(x, \xi)\right| \leq C_{\alpha, \beta}\langle x\rangle^{m-|\alpha|}\langle\xi\rangle^{\mu-|\beta|}
$$

and $\mathcal{S}^{m}=\cap_{\mu \in \mathbb{Z}} \mathcal{S}^{m}(\mu)$. We set $P=\mathrm{Op}(p(x, \xi))=p(x, D)$ for the PDO with symbol $p \in \mathcal{S}^{m}$ defined for $u \in \mathcal{S}\left(\mathbb{R}^{3}\right)$ by

$$
(P u)(x)=\int_{\mathbb{R}^{3}} e^{i<x, \xi>} p(x, \xi) \widehat{u}(\xi) \frac{d \xi}{(2 \pi)^{3 / 2}},
$$

where $\widehat{u}$ denotes the Fourier transform of $u$

$$
\widehat{u}(\xi)=\int_{\mathbb{R}^{3}} e^{-i<x, \xi>} u(x) \frac{d x}{(2 \pi)^{3 / 2}} .
$$

With this notations an operator with symbol $p \in \mathcal{S}^{m}$ is bounded (compact) if $m \leq 0(m<0)$. Now we are able to define the identifications $J_{ \pm}$.

Lemma 2.1 Let us fix $\lambda \in(0,+\infty), r \in(0, \lambda / 2)$, and let $\psi, \eta \in C^{\infty}\left(\mathbb{R}^{3},[0,1]\right)$ be cut-off functions satifying :

i) $\eta(x)=0$ if $|x| \leq R$ and $\eta(x)=1$ if $|x| \geq R+1$,

ii) $\psi(\xi)=1$ if ||$\xi|-\lambda| \leq r$ and $\psi(\xi)=0$ if ||$\xi|-\lambda| \geq 2 r$.

For any choice of functions $\eta$ and $\psi$ we set $J_{ \pm}=\mathrm{Op}\left(j_{ \pm}(x, \xi)\right)$ where

$$
j_{ \pm}(x, \xi)=e^{i \Phi_{ \pm}(x, \xi)} \eta(x) \psi(\xi) .
$$

Then $j_{ \pm} \in \mathcal{S}^{0}$ and $J_{ \pm}$is a bounded operator in $\mathbb{L}^{2}\left(\mathbb{R}^{3}\right)$. Additionally the symbol of the effective perturbation $T_{ \pm}=H J_{ \pm}-J_{ \pm} H_{0}=\mathrm{Op}\left(t_{ \pm}(x, \xi)\right)$ belongs to $\mathcal{S}^{m}$ for all $m \in \mathbb{Z}$.

Proof: Since, by (2.4),$\Phi_{ \pm}(x, \xi)$ is an homogeneous function of $x$ and $\xi$ (for $|x| \geq R$ ) of degree 0 , it satisfies in the whole phase-space the estimate :

$$
\left|\partial_{x}^{\alpha} \partial_{\xi}^{\beta} \Phi_{ \pm}(x, \xi)\right| \leq C_{\alpha, \beta}|x|^{-|\alpha|}|\xi|^{-|\beta|}, \quad \forall \alpha, \beta \in \mathbb{N}^{3}, \quad|x| \geq R .
$$

Thus taking into account the definition of cut-off functions $\eta$ and $\psi$ we get that $j_{ \pm} \in \mathcal{S}^{0}$ and $J_{ \pm}$is bounded by the Calderon-Vaillancourt Theorem. Now we calculate the symbol of the effective perturbation

$$
t_{ \pm}(x, \xi)=e^{-i<x, \xi>}\left(H-|\xi|^{2}\right) e^{i<x, \xi>} j_{ \pm}(x, \xi) .
$$


Using (2.1), (2.7), and that $\nabla_{x} \Phi_{ \pm}(x, \xi)=A(x)$, by (2.5), we get

$$
t_{ \pm}(x, \xi)=e^{i \Phi_{ \pm}(x, \xi)}\left(-2 i<\xi, \nabla_{x} \eta(x)>-\Delta_{x} \eta(x)\right) \psi(\xi) .
$$

Thus $t_{ \pm}$has a compact support in $x$ and $\xi$ and belongs to $\mathcal{S}^{m}$ for all $m \in \mathbb{Z}$. $\square$

2.2. Our proof of the existence and asymptotic completeness of modified wave operators (1.6) is based on the scheme of smooth perturbations. Then it relies on the well-known limiting absorption principle :

Theorem 2.2 (Limiting Absorption Principle) Let $H$ be the operator (1.5) with potential (1.1). Then, for all bounded interval $\Lambda \subset(0, \infty)$, disjoint from 0 , the operator function $\langle x\rangle^{-s} R(z)^{n}\langle x\rangle^{-s}, s>n-1 / 2$, is (Hölder-) continuous in norm in the region $\operatorname{Re}(z) \in \Lambda, \pm \operatorname{Im}(z) \in(0,1]$ and

$$
\sup _{\substack{\operatorname{Re} z \in \Lambda \\ 1 \geq|\operatorname{Im} z|>0}}\left\|\langle x\rangle^{-s} R(z)^{n}\langle x\rangle^{-s}\right\| \leq c, \quad \forall s>n-1 / 2 .
$$

In particular the spectrum of $H$ in $\Lambda$ is absolutely continuous and the operators $\langle x\rangle^{-s}, s>1 / 2$ are $H$-smooth on $\Lambda$ (in the sense of Kato).

This result can easily be derived from the Mourre commutator method Jen85 and the absence of positive eigenvalues for the operator (1.5) IU71. Now, since $t_{ \pm}$is short-range in the whole space, our proof of existence and completeness of wave operators relies only on the theorem 2.2 in contrast to RY02b] where the radiation estimate was also needed.

Proposition 2.3 Let $E$ and $E_{0}$ be, respectively, the spectral mesure of $H$ and $H_{0}$, and $J_{ \pm}$be constructed as under the assumptions i) and ii) of lemma 2.1] Set $\Lambda=(\lambda-r, \lambda+r)$ then the wave operators $W_{ \pm}\left(H, H_{0}, J_{ \pm}\right)$and $W_{ \pm}\left(H_{0}, H, J_{ \pm}^{*}\right)$ exist, are isometric on, respectively, $E_{0}(\Lambda)$ and $E(\Lambda)$ and are adjoint one to each other. Additionally asymptotic completeness holds for the triple $\left(H, H_{0}, J_{ \pm}\right)$, that is

$$
\begin{aligned}
\operatorname{Ran}\left(W_{ \pm}\left(H, H_{0}, J_{ \pm}\right) E_{0}(\Lambda)\right) & =\operatorname{Ran}(E(\Lambda)) \\
\operatorname{Ran}\left(W_{ \pm}\left(H_{0}, H, J_{ \pm}^{*}\right) E(\Lambda)\right) & =\operatorname{Ran}\left(E_{0}(\Lambda)\right) .
\end{aligned}
$$

Proof: Since the operators $\langle x\rangle^{-s}$ are $H$ and $H_{0}$-smooth for all $s>1 / 2$, the effective perturbation admits a decomposition into a product of smooth perturbations

$$
T_{ \pm}=\langle x\rangle^{-1}\left(\langle x\rangle T_{ \pm}\langle x\rangle\right)\langle x\rangle^{-1}
$$


because the PDO $\langle x\rangle T_{ \pm}\langle x\rangle$ belong to $\mathcal{S}^{m}$ for all $m \in \mathbb{Z}$ and so is a bounded operator. This is sufficient to prove the existence of $W_{ \pm}\left(H, H_{0}, J_{ \pm}\right)$and $W_{ \pm}\left(H_{0}, H, J_{ \pm}^{*}\right)$ which are obviously adjoint one to each other. Now, by the chain rule, isometricity and completeness of $W_{ \pm}\left(H, H_{0}, J_{ \pm}\right)$are, respectively, equivalent to

$$
\begin{aligned}
W_{ \pm}\left(H_{0}, H, J_{ \pm}^{*}\right) W_{ \pm}\left(H, H_{0}, J_{ \pm}\right) E_{0}(\Lambda) & =W_{ \pm}\left(H_{0}, H_{0}, J_{ \pm}^{*} J_{ \pm}\right) E_{0}(\Lambda)=E_{0}(\Lambda) \\
W_{ \pm}\left(H, H_{0}, J_{ \pm}\right) W_{ \pm}\left(H_{0}, H, J_{ \pm}^{*}\right) E(\Lambda) & =W_{ \pm}\left(H, H, J_{ \pm} J_{ \pm}^{*}\right) E(\Lambda)=E(\Lambda)
\end{aligned}
$$

The operator $J_{ \pm}^{*} J_{ \pm}-\psi^{2}(D)$ is compact since its principal symbol, equals to $(\eta(x)-1) \psi^{2}(\xi)$, is compactly supported in $x$. Together with the identity $\psi^{2}(D) E_{0}(\Lambda)=E_{0}(\Lambda)$ this leads to

$$
\begin{aligned}
W_{ \pm}\left(H_{0}, H_{0}, J_{ \pm}^{*} J_{ \pm}\right) E_{0}(\Lambda) & =W_{ \pm}\left(H_{0}, H_{0}, \psi^{2}(D) E_{0}(\Lambda)\right) \\
& =W_{ \pm}\left(H_{0}, H_{0}, E_{0}(\Lambda)\right)=E_{0}(\Lambda) .
\end{aligned}
$$

Then, $W_{ \pm}\left(H, H_{0}, J_{ \pm}\right)$are isometric. Asymptotic completeness goes on the same way remarking also that $E(\Lambda)-E_{0}(\Lambda)$ is compact.

Finally let us check that the wave operators constructed here coincide with the usual ones constructed in [LT87.

Proposition 2.4 Under assumptions i) and ii) of lemma 2.1 we have

$$
W_{ \pm}\left(H, H_{0}, J_{ \pm}\right)=W_{ \pm}\left(H, H_{0}, I d\right) \psi(D)
$$

for any choice of functions $\eta$ and $\psi$.

Proof: The proof relies on the stationary phase formula applied to the integral

$$
\left(J_{ \pm} e^{-i t H_{0}} u\right)(x)=\int_{\mathbb{R}^{3}} e^{i<x, \xi>-i t|\xi|^{2}} j_{ \pm}(x, \xi) \widehat{u}(\xi) \frac{d \xi}{(2 \pi)^{3 / 2}}
$$

Since the stationary points $\xi_{0}=\xi_{0}(t)$ of this integral are $\xi_{0}=x /(2 t)$ we get, for $u \in \mathcal{S}\left(\mathbb{R}^{3}\right)$, the asymptotics

$$
\left(J_{ \pm} e^{-i t H_{0}} u\right)(x)=\frac{e^{\mp i d \pi / 4}}{(2 t)^{d / 2}} e^{i|x|^{2} /(2 t)+i \Phi_{ \pm}(x, x /(2 t))} \widehat{u}(x /(2 t)) \eta(x) \psi(x /(2 t))+\mathrm{r}_{ \pm}(x, t)
$$


where $\mathrm{r}_{ \pm}(x, t)$ tends to 0 in $\mathbb{L}^{2}\left(\mathbb{R}^{3}\right)$ as $t \rightarrow \pm \infty$. Now, by (2.4), we have $\Phi_{ \pm}(x, x /(2 t))=0$ for $|x| \geq R$ so the phase factor $\exp \left(i \Phi_{ \pm}(x, x /(2 t))\right)$ is inessential and

$$
\lim _{t \rightarrow \pm \infty}\left(J_{ \pm} e^{-i t H_{0}}-e^{-i t H_{0}}\right) u=\lim _{t \rightarrow \pm \infty}(\eta-1) e^{-i t H_{0}} \psi(D) u=0
$$

since $\eta-1$ is $H_{0}$-compact. In conclusion usual wave operators exists and coincide with the wave operators of proposition 2.3 .

Remark 2.5 If a "short-range" electromagnetic perturbation $\left(V_{0}, A_{0}\right)$

$$
\left|V_{0}(x)\right|+\left|A_{0}(x)\right|+\left|\operatorname{div} A_{0}(x)\right| \leq C\langle x\rangle^{-\rho}, \quad \rho>1,
$$

is added to the operator $H$, then all the results of this section remain true without changing the definition (2.7) of identifications $J_{ \pm}$. The additional

term $\widetilde{T}_{ \pm}$arising in the effective perturbation $T_{ \pm}$are short-range. Since $\rho>1$, and by theorem 2.2. they admit a factorization into a product of $H$-smooth operators similar to (2.9)

$$
\widetilde{T}_{ \pm}=\langle x\rangle^{-\rho / 2}\left(\langle x\rangle^{\rho / 2} \widetilde{T}_{ \pm}\langle x\rangle^{\rho / 2}\right)\langle x\rangle^{-\rho / 2} .
$$

\section{The scattering matrix}

In this section we consider the SM for the pair $H, H_{0}$ and its stationary representation. We do not give a proof of formula (1.7) (a complete justification can be found in [Yaf00]), but we rewrite it into a sum of bounded operators on $\mathbb{L}^{2}\left(\mathbb{S}^{2}\right)$ which gives its precise meaning to the formula (1.7). Thus we can make the analysis of spectral properties and singularities of $S(\lambda)$ for all $\lambda>0$.

Let us decompose formula (1.7) as follow

$$
\begin{aligned}
S(\lambda) & =\mathcal{W}(\lambda)+S_{1}(\lambda)+S_{2}(\lambda) \\
S_{1}(\lambda) & =-2 i \pi \Gamma_{0}(\lambda) J_{+}^{*} T_{-} \Gamma_{0}^{*}(\lambda) \\
S_{2}(\lambda) & =2 i \pi \Gamma_{0}(\lambda) T_{+}^{*} R(\lambda+i 0) T_{-} \Gamma_{0}^{*}(\lambda)
\end{aligned}
$$

with $\mathcal{W}(\lambda)$ and $\Gamma_{0}(\lambda)$ given by (1.8) and (1.9). In the following three propositions we analyze separately the terms $\mathcal{W}(\lambda), S_{1}(\lambda)$ and $S_{2}(\lambda)$. 
Proposition 3.1 The operator $\mathcal{W}(\lambda)$ defined by (1.8) is the operator of multiplication by the function $\mathrm{w}(\omega)$ defined on $\mathbb{S}^{2}$ by (1.10).

Proof: First remark that the commutator

$$
\left[H_{0}, J_{+}^{*} J_{-}\right]=T_{+}^{*} J_{-}+J_{+}^{*} T_{-}
$$

admits a factorization into a sum of products of $H_{0}$-smooth operators. Then the wave operator $W_{+}\left(H_{0}, H_{0}, J_{+}^{*} J_{-}\right)$is well defined, commutes with $H_{0}$ (by the interwinning property), and so it reduces to multiplication by the operator valued function $\mathcal{W}(\lambda)$ in the spectral representation of $H_{0}$. Up to compact terms the operator $J_{+}^{*} J_{-}$is the PDO with principal symbol $\exp (i \Theta(x, \xi))$ with

$$
\Theta(x, \xi)=\Phi_{-}(x, \xi)-\Phi_{+}(x, \xi),
$$

taking into account (2.4) we obtain that $\Theta$ does not depends on $x$

$$
\Theta(x, \xi)=\int_{\varphi_{-\xi}}^{\varphi_{x}} a(\varphi) d \varphi-\int_{\varphi_{\xi}}^{\varphi_{x}} a(\varphi) d \varphi=\int_{\varphi_{-\xi}}^{\varphi_{\xi}} a(\varphi) d \varphi=: \Theta(\xi) .
$$

Now since the operator $\exp (i \Theta(D))$ commutes with $H_{0}$ we get

$$
\begin{aligned}
W_{+}\left(H_{0}, H_{0}, J_{+}^{*} J_{-}\right) E_{0}(\Lambda) & =s-\lim _{t \rightarrow \pm \infty} e^{-i t H_{0}} J_{+}^{*} J_{-} e^{-i t H_{0}} E_{0}(\Lambda) \\
& =s-\lim _{t \rightarrow \pm \infty} e^{-i t H_{0}} e^{i \Theta(D)} e^{-i t H_{0}} E_{0}(\Lambda) \\
& =e^{i \Theta(D)} E_{0}(\Lambda) .
\end{aligned}
$$

The function $\Theta$ is obviously homogeneous of degree 0 , by (3.4), Together with the obvious identity $\varphi_{-\omega}=\pi-\varphi_{\omega}$ this leads to $\exp (i \Theta(\sqrt{\lambda} \omega))=$ $\exp (i \Theta(\omega))=\mathrm{w}(\omega)$. Then in the spectral representation where $H_{0}$ is diagonal the operator $\mathcal{W}(\lambda)$ reduces to the operator of multiplication by the function (1.10).

Remark 3.2 From the physical point of view, $\Theta(x, \xi)$ is the circulation of the magnetic potential $A(x)$ along the "closed" contour symbolized by doted lines on Figure 1. In particular the calculation of function $\Theta$ is independent of the gauge choosen for $A(x)$. Thus the scheme developed before applies to any magnetic potential $\tilde{A}(x)$ satisfying $\operatorname{curl}(\tilde{A})=\operatorname{curl}(A)$, however usual wave operators $W_{ \pm}\left(\tilde{H}, H_{0}\right)$, with $\tilde{H}=(D-\tilde{A})^{2}$, should not exists if the transversal gauge is not assumed. 
Here we note that the kernel of $\mathcal{W}(\lambda)$ is $\mathrm{w}(\omega) \delta\left(\omega, \omega^{\prime}\right)$ where $\delta$ denotes the Dirac distribution on $\mathbb{S}^{2}$. Below we show that the kernel of $S(\lambda)$ does not contains any other singularity.

Proposition 3.3 The operator $S_{1}(\lambda)$, defined in (3.2), is an integral operator on $\mathbb{S}^{2}$ with a smooth kernel $\mathrm{s}_{1}\left(\omega, \omega^{\prime} ; \lambda\right) \in C^{\infty}\left(\mathbb{S}^{2} \times \mathbb{S}^{2} \times \mathbb{R}_{+}^{*}\right)$. In particular $S_{1}(\lambda)$ belongs to the Hilbert-Schmidt class.

Proof: By equations (1.9), (2.6) , the operator $S_{1}(\lambda)=-2 i \pi \Gamma_{0}(\lambda) J_{+}^{*} T_{-} \Gamma_{0}^{*}(\lambda)$ is the restriction of a PDO on $\mathbb{L}^{2}\left(\mathbb{R}_{\xi}^{3}\right)$ with amplitude $\overline{j_{+}(x, \xi)} t_{-}\left(x, \xi^{\prime}\right)$ to the sphere $|\xi|^{2}=\left|\xi^{\prime}\right|^{2}=\lambda$, thus it is an integral operator on $\mathbb{L}^{2}\left(\mathbb{S}^{2}\right)$ with kernel

$$
\mathrm{s}_{1}\left(\omega, \omega^{\prime} ; \lambda\right)=-\frac{i \sqrt{\lambda}}{8 \pi^{2}} \int_{\mathbb{R}^{3}} e^{i \sqrt{\lambda}<\omega^{\prime}-\omega, x>} \overline{j_{+}(x, \sqrt{\lambda} \omega)} t_{-}\left(x, \sqrt{\lambda} \omega^{\prime}\right) d x .
$$

Since the amplitude $\overline{j_{+}(x, \sqrt{\lambda} \omega)} t_{-}\left(x, \sqrt{\lambda} \omega^{\prime}\right)$ is compactly supported in $x$ (due to the presence of derivatives of function $\eta$ defined in lemma 2.1) the integral above obviously converge. Differentiating expression (3.5) we get that $\mathrm{s}_{1}\left(\omega, \omega^{\prime} ; \lambda\right)$ is a $C^{\infty}$-function. In particular $\left|\mathrm{s}_{1}\left(\omega, \omega^{\prime} ; \lambda\right)\right|^{2}$ is bounded and the Hilbert-Schmidt norm

$$
\int_{\mathbb{S}^{2}} \int_{\mathbb{S}^{2}}\left|\mathrm{~s}_{1}\left(\omega, \omega^{\prime} ; \lambda\right)\right|^{2} d \omega d \omega^{\prime}
$$

of $S_{1}(\lambda)$ is finite.

Proposition 3.4 The operator $S_{2}(\lambda)$, defined in (3.3), is an integral operator on $\mathbb{S}^{2}$ with a smooth kernel $\mathrm{s}_{2}\left(\omega, \omega^{\prime} ; \lambda\right) \in C^{\infty}\left(\mathbb{S}^{2} \times \mathbb{S}^{2} \times \mathbb{R}_{+}^{*}\right)$. $S_{2}(\lambda)$ belongs to the Hilbert-Schmidt class.

Proof: Let $\psi_{0}(x, \xi)=\exp (i<\xi, x>)$, then the kernel of the operator $S_{2}(\lambda)=2 i \pi \Gamma_{0}(\lambda) T_{+}^{*} R(\lambda+i 0) T_{-} \Gamma_{0}^{*}(\lambda)$ is formaly defined by the expression

$$
\mathrm{s}_{2}\left(\omega, \omega^{\prime} ; \lambda\right)=\frac{i \sqrt{\lambda}}{8 \pi^{2}}\left(T_{+}^{*} R(\lambda+i 0) T_{-} \psi_{0}\left(\cdot, \sqrt{\lambda} \omega^{\prime}\right), \psi_{0}(\cdot, \sqrt{\lambda} \omega)\right)_{\mathbb{L}^{2}\left(\mathbb{R}^{3}\right)} .
$$


Formula (3.6) is automatically justified if its right hand side is a continuous function of $\omega, \omega^{\prime}, \lambda$. The derivatives $\partial_{\omega}^{\alpha} \partial_{\omega^{\prime}}^{\alpha^{\prime}} \partial_{\lambda}^{m} \mathrm{~S}_{2}\left(\omega, \omega^{\prime} ; \lambda\right)$ consist in a sum of terms of the form

$$
\begin{aligned}
& \left(T_{+}^{*} R^{n}(\lambda+i 0) T_{-}\langle x\rangle^{\beta^{\prime}} \psi_{0}\left(\cdot, \sqrt{\lambda} \omega^{\prime}\right),\langle x\rangle^{\beta} \psi_{0}(\cdot, \sqrt{\lambda} \omega)\right)_{\mathbb{L}^{2}\left(\mathbb{R}^{3}\right)} \\
& =\left(\langle x\rangle^{-n} R^{n}(\lambda+i 0)\langle x\rangle^{-n} Q_{-}\langle x\rangle^{-2} \psi_{0}\left(\cdot, \sqrt{\lambda} \omega^{\prime}\right), Q_{+}\langle x\rangle^{-2} \psi_{0}(\cdot, \sqrt{\lambda} \omega)\right)_{\mathbb{L}^{2}\left(\mathbb{R}^{3}\right)}
\end{aligned}
$$

with $Q_{+}=\langle x\rangle^{n} T_{+}\langle x\rangle^{\beta+2}, Q_{-}=\langle x\rangle^{n} T_{-}\langle x\rangle^{\beta^{\prime}+2} \in \mathcal{S}^{m}$ for all $m \in \mathbb{Z}$ and $|\alpha|+\left|\alpha^{\prime}\right|+m=|\beta|+\left|\beta^{\prime}\right|+n$. Since the operators $Q_{ \pm}$and $\langle x\rangle^{-n} R^{n}(\lambda+$ i0) $\langle x\rangle^{-n}$ are bounded on $\mathbb{L}^{2}\left(\mathbb{R}^{3}\right)$ (by theorem 2.2) and taking into account that $\langle x\rangle^{-2} \psi_{0}(\cdot, \xi) \in \mathbb{L}^{2}\left(\mathbb{R}^{3}\right)$ those expressions are correctly defined and bounded. Finally, since $\psi_{0}(\cdot, \sqrt{\lambda} \omega),\langle x\rangle^{-n} R^{n}(\lambda+i 0)\langle x\rangle^{-n}$ are continuous in $\lambda$ and $\omega$, we have shown that $\mathrm{s}_{2}\left(\omega, \omega^{\prime} ; \lambda\right)$ is a $C^{\infty}$-function. In particular $\left|\mathrm{s}_{2}\left(\omega, \omega^{\prime} ; \lambda\right)\right|^{2}$ is bounded and the Hilbert-Schmidt norm of $S_{2}(\lambda)$ is finite.

Combining propositions 3.13 .3 and 3.4 we obtain

Theorem 3.5 Let $H$ be the operator (1.5) with potential (1.1), $S(\lambda)$ be the $S M$ for the pair $H, H_{0}=-\Delta$ and $\mathcal{W}(\lambda)$ be the operator of multiplication on $\mathbb{S}^{2}$ by the function $\mathrm{w}$ defined in (1.10). Then the operator $S(\lambda)-\mathcal{W}(\lambda)$ has an infinitly-smooth kernel, in particular it belongs to the Hilbert-Schmidt class.

We can now prove the two essentials results on spectral properties of the SM for the pair $H, H_{0}$.

Theorem 3.6 Let $H$ be the operator (1.5) with potential (1.1) and $S(\lambda)$ be the $S M$ for the pair $H, H_{0}=-\Delta$, then the essential spectrum of $S(\lambda)$ is given by (1.11).

Proof: Since $\mathcal{W}(\lambda)$ is the operator of multiplication by w, its (continuous) spectrum coincides with the range of the function w. Since the function $a$ in (1.1) is positive and taking into account relation (1.4) the range of the function (3.4) equals the interval $[-\phi, \phi]$ and the spectrum of $\mathcal{W}(\lambda)$ is the image of this interval by the function $v \mapsto \exp (i v)$. Finally, since $S(\lambda)-\mathcal{W}(\lambda)$ is Hilbert-Schmidt, and also compact, thank's to Weyl theorem the essential spectrum of $S(\lambda)$ coincides with the essential spectrum of $\mathcal{W}(\lambda)$ that is (1.11). 
Theorem 3.7 Let $H$ be the operator (1.5) with potential (1.1) and $S(\lambda)$ be the $S M$ for the pair $H, H_{0}=-\Delta$, then the total scattering cross-section $\Sigma_{\text {tot }}\left(\omega_{0} ; \lambda\right)$ (defined by (1.12) and (1.13) $)$ is finite for all incident direction $\omega_{0}$.

Proof: The kernel of the principal part $\mathcal{W}(\lambda)$ is $\mathrm{w}(\omega) \delta\left(\omega, \omega^{\prime}\right)$ where $\delta$ denotes the Dirac distribution on $\mathbb{S}^{2}$. In particular its support is concentrated on the diagonal $\omega=\omega^{\prime}$. Off the diagonal the kernel of $S(\lambda)$ reduces to the sum $\mathrm{s}_{1}\left(\omega, \omega^{\prime} ; \lambda\right)+\mathrm{s}_{2}\left(\omega, \omega^{\prime} ; \lambda\right)$. Since $\mathrm{s}_{1}$ and $\mathrm{s}_{2}$ are infinitly smooth functions the integral (1.13) converge and the total scattering cross-section is finite for all $\omega_{0}$.

Remark 3.8 The result of theorem 3.7 is conserved under short-range perturbations $\left(V_{0}, A_{0}\right)$ if we suppose that (2.10) is satisfied for some $\rho>3$.

\section{The Aharonov-Bohm effect in dimension 3}

Finally, we want to make some remarks on the Aharonov-Bohm effect. Since the 3 dimensionnal example of magnetic field treated here is compactly supported it is somewhat natural to compare our results to those obtained in the 2 dimensionnal case. Let the Aharonov-Bohm Hamiltonian be the operator $H_{A B}=\left(D-A_{A B}(x)\right)^{2}$, on $\mathbb{L}^{2}\left(\mathbb{R}^{2}\right)$, with magnetic potential

$$
A_{A B}(x)=a\left(\theta_{x}\right) \frac{\left(-x_{2}, x_{1}\right)}{|x|^{2}}, \quad|x| \geq R>0,
$$

where $a \in C^{\infty}(\mathbb{R})$ is a $2 \pi$-periodic function of the polar angle $\theta_{x}$ associated to $x=\left(x_{1}, x_{2}\right)$. The family of potentials satisfying (4.1) includes all compactly supported magnetic field in dimension 2. For potential (4.1) an analysis, similar to the one made here, was developed in [RY02a. With the notations

$$
\phi_{A B}=\int_{0}^{2 \pi} a(\vartheta) d \vartheta, \quad f(\theta)=\int_{\theta}^{\theta+\pi} a(\vartheta) d \vartheta
$$

it is shown that for $S_{A B}(\lambda)$, the $\mathrm{SM}$ associated to the pair $H_{A B}, H_{0}$, we have that

$$
\sigma_{e s s}\left(S_{A B}(\lambda)\right)=\exp (i f(\mathbb{R})) \cup \exp (-i f(\mathbb{R}))
$$


and the differential scattering cross-section admits the asymptotic

$$
\Sigma_{d i f f}\left(\omega, \omega_{0} ; \lambda\right)=\frac{1}{2 \pi \sqrt{\lambda}} \frac{\sin ^{2}\left(\phi_{A B} / 2\right)}{\sin ^{2}(\theta / 2)}+\mathcal{O}\left(\frac{\ln (\theta)}{\theta}\right),
$$

as $\omega \rightarrow \omega_{0}$, with $\left|\omega-\omega_{0}\right|=2 \sin (\theta / 2)$. The equations (4.2) and (4.3) generalize the results obtained by various authors (see Rui83]) for the potential (4.1) with $a=C^{\text {ste }}\left(=\phi_{A B} /(2 \pi)\right)$ :

$$
\sigma\left(S_{A B}(\lambda)\right)=\sigma_{p p}\left(S_{A B}(\lambda)\right)=\left\{e^{i \phi_{A B} / 2}, e^{-i \phi_{A B} / 2}\right\}
$$

and

$$
\Sigma_{\text {diff }}^{A B}\left(\omega, \omega_{0} ; \lambda\right)=\frac{1}{2 \pi \sqrt{\lambda}} \frac{\sin ^{2}\left(\phi_{A B} / 2\right)}{\sin ^{2}(\theta / 2)} .
$$

If we compare (1.11) to (4.2) we remark that both SM have intermediary spectral properties between the general cases of short and long-range potentials where, respectively, the essential spectrum reduce to $\{1\}$ or covers the whole unit circle (see [RY02b]). From (4.3) we see that in dimension 2 the total scattering cross-section is infinite except if the magnetic flux $\phi_{A B} \in 2 \pi \mathbb{Z}$, on the contrary for potential (1.1), in dimension 3, this situation does not appear.

In contrast to the 2-dimensionnal case, only few authors have been interested in the 3 dimensionnal case [Tam95, BR01, so the potential (1.1) can be regarded as an interesting example. Since the family of potentials satisfying (1.1) does not contains all compactly supported magnetic fields in dimension 3 we could not exclude the existence of such fields with infinite total cross-section, but it seems that the situation described in this paper is very general. Indeed, let us consider a magnetic potential $A$ obtained from an arbitrary comptacly supported magnetic field that is

$$
\operatorname{curl} A(x)=0 \Longleftrightarrow A(x)=\nabla \Phi(x)
$$

for large $|x|$ and some regular function $\Phi$. It is quite plausible that we can choose $\Phi=\Phi_{ \pm}$, the solutions of the eikonal equation (2.2), as in the case of potential (1.1). If this conjecture is verified then we can generalize the scheme developped here to all potentials satisfiying (4.4). As in section 2 we can defined the wave operators of lemma 2.1 which coincide with the usual one and thus are complete. Similarly the results of section 3 would be 
generalized. Remarking that the results of propositions 3.3 and 3.4 do not depend on the phases $\Phi_{ \pm}$and so they holds for the arbitrary potential $A$. Thus all the singularities of the SM are contained in the term $\mathcal{W}(\lambda)$ defined by (1.8). As shown in the proof of proposition 3.1 the singularities of $\mathcal{W}(\lambda)$ reduce to the Dirac singularity if the function $\Theta(x, \xi)=\Phi_{-}(x, \xi)-\Phi_{+}(x, \xi)$ is independent of $x$ (for large $|x|$ ). This fact will follows from the initial conjecture since

$$
\nabla_{x} \Theta(x, \xi)=\nabla_{x} \Phi_{-}(x, \xi)-\nabla_{x} \Phi_{+}(x, \xi)=A(x)-A(x)=0 .
$$

Then for any compactly supported magnetic field the SM will reduce to multiplication by the function $\exp (i \Theta(\omega))$, up to a $C^{\infty}$-kernel operator. So in contrast to the 2 dimensional case, the total scattering cross section will always be finite in dimension 3. A final argument for this conjecture can be found in Yaf02. In this paper Yafaev shown a result similar to the one conjectured here, but for short range magnetic potentials, that is the high energy limit of the SM is the operator of multiplication by $\exp \left(i \int_{\mathbb{R}}<\right.$ $A(t \omega, \omega) d t)$.

\section{References}

[AB59] Y. Aharonov and D. Bohm. Significance of electromagnetic potentials in the quantum theory. Phys. Rev. (2), 115:485-491, 1959.

[BR01] J. M. Bily and D. Robert. The semi-classical van-vleck formula. application to the Aharonov-Bohm effect. Preprint, 2001.

[IU71] Teruo Ikebe and Jun Uchiyama. On the asymptotic behavior of eigenfunctions of second-order elliptic operators. J. Math. Kyoto Univ., 11:425-448, 1971.

[Jen85] Arne Jensen. Propagation estimates for Schrödinger-type operators. Trans. Amer. Math. Soc., 291(1):129-144, 1985.

[LT87] Michael Loss and Bernd Thaller. Scattering of particles by longrange magnetic fields. Ann. Physics, 176(1):159-180, 1987.

[Nic94] François Nicoleau. Matrices de diffusion pour l'opérateur de Schrödinger en présence d'un champ magnétique. Phénomène de 
Aharonov-Bohm. Ann. Inst. H. Poincaré Phys. Théor., 61(3):329346, 1994.

[Rui83] S. N. M. Ruijsenaars. The Aharonov-Bohm effect and scattering theory. Ann. Physics, 146(1):1-34, 1983.

[RY02a] Ph. Roux and D. R. Yafaev. On the mathematical theory of the Aharonov-Bohm effect. J. Phys. A, 35, 2002.

[RY02b] Ph. Roux and D. R. Yafaev. The scattering matrix for the Schrödinger operator with a long-range electromagnetic potential. Preprint, 2002.

[Tam95] H. Tamura. Shadow scattering by magnetic fields in two dimensions. Ann. Inst. H. Poincaré Phys. Théor., 63(3):253-276, 1995.

[Tay81] Michael E. Taylor. Pseudodifferential operators. Princeton University Press, Princeton, N.J., 1981.

[Yaf98] D. R. Yafaev. The scattering amplitude for the Schrödinger equation with a long-range potential. Comm. Math. Phys., 191(1):183218, 1998.

[Yaf00] Dmitri Yafaev. Scattering theory: some old and new problems. Springer-Verlag, Berlin, 2000.

[Yaf02] D. R. Yafaev. High energy and smoothness asymptotic expansion of the scattering amplitude. preprint, 2002. to appear in J. Funct. Anal. 